\title{
Archéologie du service public audiovisuel : quel passé pour quel futur ?
}

Article inédit. Mis en ligne le 23 décembre 2013.

\section{Serge Regourd}

Serge Regourd est Professeur à l'Université Toulouse 1-Capitole où il dirige l'Institut du droit de la culture et de la communication (IDETCOM, EA 785). Il dirige aussi plusieurs masters 2 : le master Droit des médias et de la communication, le master Communication et administration des activités culturelles. Il est l'auteur de nombreuses publications concernant les médias audiovisuels, notamment les ouvrages "La télévision des Européens" (La Doc. Française 1992), "Droit de la communication audiovisuelle" (PUF 2001), "L'Exception culturelle" (PUF 2004), "Vers la fin de la télévision publique ? "(Ed. de L'Attribut 2009). Il a été expert auprès de diverses institutions et organisations professionnelles de l'audiovisuel.

Plan

Le service public englué dans la matrice politique

La velléité de "rupture du cordon ombilical»

La diversification des figures de la dépendance politique

Le service public confronté à la dynamique marchande

Privatisation de TF 1 et récusation de la notion de service public

La menace de délitement du service public

Conclusion

Références

\section{Résumé}

L'archéologie du service public audiovisuel révèle une singularité du cas français, dominé par un certain nombre de "malédictions" à la fois politiques et économiques. Sur le terrain politique, la soumission de la radio-télévision française au pouvoir Exécutif s'est notamment traduite par le concept de "Voix de la France", et l'affirmation à partir de 1982 de la volonté de couper ce "cordon ombilical" s'est avérée d'application particulièrement tourmentée. Sur le terrain économique, la privatisation de TF1 en 1986 constitue une illustration sans aucun équivalent dans les autres Etats européens, du triomphe d'une nouvelle rationalité marchande, exprimant corrélativement une grave sous-évaluation des enjeux inhérents à la place du service public. L'entrée dans l'ère numérique souffre d'un certain nombre de ces hypothèques héritées du passé.

Mots-clés : Radio, télévision, Etat, marché, régulation, dépendance politique

\section{Abstract}

Archeology of public service broadcasting

The archeology of public service broadcasting reveals a peculiarity of French situation, dominated by some "curses" on both political and economic aspects. In the political sphere, the dependence of the French radio and television relative to the executive power was reflected in particular by the concept of "Voice of France", and the predications about independent public service in 1982 have been difficult to substantiate. In the economic sphere, the privatization of TF1 in 1986 is an illustration - with no equivalent in other European countries - of the triumph of a new market rationality, expressing correspondingly severe undervaluation of issues inherent in the place of public service. Entering the digital age suffers from a number of these problems inherited. Keywords: Radio, Television, state, market, regulation, political dependence 


\section{Resumen}

Arqueología de la radiodifusión de servicio público

La arqueología de la radiodifusión de servicio público revela una peculiaridad del caso francés, dominado por una serie de "maldiciones" en tanto política como económica. En el ámbito político, la presentación de la radio y la televisión francesa en el poder ejecutivo se reflejó en particular, por el concepto de "La Voz de Francia", y puestos en el servicio público independiente en 1982 fueron difíciles de justificar. En el ámbito económico, la privatización de TF1 en 1986 es un ejemplo - con no equivalente en otros países europeos del triunfo de una nueva racionalidad del mercado, que expresan infravaloración correspondientemente grave de los problemas inherentes al servicio público. Al entrar en la era digital, la radio-television adolece de una serie de estos problemas heredados.

Palabras clave: Radio, TV, Estado, mercado, ldependencia política, regulación

\section{INTRODUCTION}

Par une décision du 11 février 2010, Mme Borvo et autres, le Conseil d'Etat a procédé à une double annulation. D'une part de la lettre de la ministre de la Culture et de la Communication du 15 décembre 2008 enjoignant au conseil d'administration de la société France-Télévisions de supprimer la publicité sur ses chaînes, en anticipation d'une loi non encore adoptée, en discussion au Sénat. D'autre part de la délibération subséquente dudit conseil d'administration, prise le lendemain, 16 décembre 2008, obtempérant à cette injonction et confiant au P-D.G de France-Télévisions, la responsabilité de procéder effectivement à la suppression de la publicité à compter du 5 janvier 2009, alors que la loi ayant un tel objet ne fut édictée que le 5 mars 2009 (1).

Cette situation - et l'annulation des décisions en cause - fournissent un concentré des malédictions qui n'ont cessé de caractériser le traitement du service public de la communication audiovisuelle depuis les débuts de la Vème République (2).

Selon une approche de type archéologique, empruntée à Michel Foucault et à son analyse de «l'archéologie du savoir» (3), l'on peut considérer que ces manières de traiter la télévision publique expriment la pérennité des effets, selon d'autres voies, de strates chronologiques antécédentes, caractérisées par la prégnance des déterminants politiques. L'épisode précité illustre la persistance des lourdes hypothèques politiques pesant, plus globalement, sur la communication audiovisuelle : la Haute Juridiction administrative n'a guère éprouvé de difficultés à annuler les actes en cause en se fondant sur la double incompétence de la Ministre et du Conseil d'Administration de France-Télévisions pour prendre des mesures qui «relèvent du domaine de la loi », comme l'avait entre-temps, rappelé le Conseil Constitutionnel dans sa décision du 3 mars 2009 (4) .

L'incompétence de la ministre et du C.A de France-Télévisions paraît, en l'occurrence, revêtir une portée plus radicale: un ministre ne dispose d'aucune compétence pour imposer une décision à une société anonyme, fût-elle chargée d'une mission de service public, et le C.A de celle-ci n'a pas à obtempérer à une telle injonction ministérielle.

Cette manière de procéder a connu des précédents tout aussi caricaturaux. Ainsi, par exemple, alors que la loi du 30 septembre 1986, était encore en discussion, et que subsistaient donc les concessions de service public prévues par la loi du 29 juillet 1982 (5), 
deux décrets du 30 juillet 1986 prononcèrent les résiliations des concessions des deux premières chaînes privées. Ces décrets furent annulés par deux arrêts d'Assemblée du Conseil d'Etat. (Mais, comme dans le cas précédemment évoqué, l'adoption de la loi, intervenue entre-temps priva ces annulations de réelle portée). Ces péripéties contentieuses illustrent, en tout cas, la pérennité des principales lignes de force qui caractérisent l'archéologie de la régulation de la communication audiovisuelle concernant l'audiovisuel public, et justifient la prise en compte de celle-ci pour éclairer le présent et penser le futur.

Dans ce domaine, en effet, des médias de masse, marqué par l'importance des mutations politiques, économiques, financières, techniques et culturelles, la connaissance et la compréhension du système de régulation de l'audiovisuel public ne sauraient se suffire du formalisme des règles applicables: l'analyse de la situation contemporaine requiert l'éclairage des strates historiques - ou archéologiques - antécédentes : celles-ci font apparaître, successivement, puis simultanément, une sur-détermination des critères politiques et économiques dans le processus de régulation de l'audiovisuel public. Dans les illustrations précédentes, la décision politique, brutale, porte conjointement, sur des enjeux financiers tels que les ressources publicitaires de la télévision publique.

L'on pourrait considérer que le service public de l'audiovisuel a connu une mutation décisive avec la suppression du monopole et l'ouverture corrélative aux opérateurs privés, résultant de la loi du 29 juillet 1982 elle-même consécutive à l'élection de François Mitterrand à la présidence de la République (6). Mais la portée de cette loi-charnière ne se manifeste elle-même qu'au regard de la prise en compte des strates antécédentes. Deux facteurs centraux caractérisent alors les réformes successives du système audiovisuel public : d'abord le déterminant, manifeste, outrageusement manifeste, du Politique, dans son acception souvent politicienne, sévissant à chaque échéance politique, présidentielle ou parlementaire et ayant, notamment, réduit le monopole public à un monopole politique du parti au pouvoir. Ensuite, le déterminant économique, plus récent, selon une acception principalement marchande et financière, conduisant à appréhender les évolutions des dispositifs juridiques comme de simples étapes d'adaptation aux logiques du «marché audiovisuel » et aboutissant à soumettre le service public lui-même à de telles logiques.

Le poids de ces deux déterminants est si fort qu'il paraît éclairer la panne d'imagination dont paraît souffrir l'actuel gouvernement, à l'heure où sont débattus, deux projets de loi, organique et ordinaire, sur le sujet. La réalité du consensus politique sur le marché audiovisuel a conduit à une réduction de l'ambition réformatrice à un simple affichage politique autour de la nomination des responsables de l'audiovisuel public en forme, pour l'essentiel, de retour au statu quo ante, sans la moindre prise en compte des difficultés spécifiques du service public.

"Comprendre les médias", pour reprendre le titre de l'ouvrage célèbre de Mac Luhan (7), s'agissant de l'audiovisuel public en France, conduit ainsi à prendre en compte d'abord sa difficile émancipation à l'égard de la matrice politique (I), et d'apprécier, ensuite, la singulière prévalence de la dynamique financière à laquelle il est confronté (II).

\section{LE SERVICE PUBLIC ENGLUE DANS LA MATRICE POLITIQUE}

Au terme de l'élection présidentielle de 1981, la loi du 29 juillet 1982 met fin au monopole public. En déclarant, dès son article $1^{\text {er }}$ que, désormais, «la communication audiovisuelle est libre", elle inaugure une nouvelle problématique de régulation du secteur, logiquement complétée par la création d'une autorité de régulation, la Haute Autorité de la Communication Audiovisuelle. Ces deux éléments-clés - suppression du monopole 
public et création d'une autorité administrative indépendante - sont supposés traduire la volonté politique du nouveau Président de la République de « couper le cordon ombilical » entre les médias audiovisuels et le Pouvoir Exécutif, qui avait caractérisé la période antécédente. Mais cette velléité d'émancipation ne paraît pas avoir empêché la pérennité de telles hypothèques politiques.

\section{La velléité de "rupture du cordon ombilical"}

Afin de prendre la mesure des fondements et des finalités de la loi de 1982, il convient, brièvement, de rappeler quelques-unes des empreintes génétiques initiales de l'audiovisuel depuis les débuts de la Vème République. Elles manifestent une fâcheuse confusion entre monopole public et monopole politique, entre radio-télévision de service public, et radiotélévision d'Etat. Ainsi, le Président Mitterrand pouvait-il stigmatiser les conséquences du « cordon ombilical » ne cessant de caractériser les relations de l'audiovisuel public avec son géniteur Etatique.

Le premier statut juridique de la R.T.F (Radio-Télévision Française) résultant de l'ordonnance du 4 février 1959 fait, enfin, de celle-ci après son absence de statut tout au long de la IVème République, une institution autonome, sous la forme d'un établissement public à caractère industriel et commercial (EPIC). Mais ce statut, est, conjointement dépouillé des caractères d'autonomie que lui confère traditionnellement le droit commun : la RTF est directement placée «sous l'autorité » du ministère de l'information, exprimant la réalité d'une relation hiérarchique. Son directeur général est nommé par décret, en Conseil des ministres, sans la moindre précision relative à la durée de son mandat, autorisant donc, juridiquement, une révocation discrétionnaire, à tout instant. Mais, surtout, la R.T.F est dépourvue de tout conseil d'administration, transférant l'essentiel du contrôle décisionnel au pouvoir Exécutif et, notamment, au ministre de l'information.

La création de l'ORTF, en 1964, marque, certes, quelques progrès avec la mise en place d'un tel conseil d'administration, mais celui-ci n'est encore doté que de faibles compétences, et le directeur général est toujours nommé sans précision quant à la durée de son mandat. C'est, d'ailleurs, après cette réforme et la création de l'ORTF par la loi du 27 juin 1964, que lors d'une conférence de presse, restée célèbre, du 2 juillet 1970, Georges Pompidou pouvait, sereinement, placidement, formuler sa conception de la télévision publique comme "Voix de la France ", justifiant, très officiellement, le contrôle politique exercé par le Pouvoir Exécutif, en contradiction frontale avec les principes d'égalité, et de pluralisme, supposés fonder le régime général des services publics.

Cette captation, et cette instrumentalisation politique, de la télévision publique, suscitèrent les virulentes critiques de la contestation de 1968 et post-1968, et les velléités subséquentes d'ouverture du gouvernement Chaban-Delmas. Mais la loi que celui-ci avait voulu intervint trop tard, le 3 juillet 1972, quelques jours après qu'il eût lui-même dû renoncer à ses fonctions. Son projet de décentralisation des structures fut un échec, et le progrès, consistant dans la détermination de la durée du mandat du directeur de l'ORTF, symboliquement promu P.D-G, fut aussitôt bafoué par le primat du Politique : le nouveau P.D-G, Arthur Conte, nommé pour trois ans, fut brutalement révoqué au bout de 16 mois à la suite d'un désaccord avec le ministre de l'Information.

Ainsi, l'audiovisuel, durant la période du monopole de service public, connaissait-il toutes les formes de domestication par le Pouvoir Exécutif... C'est bien une rupture avec de telles pratiques - «rupture du cordon ombilical» - que postulait la loi du 29 juillet 1982, inaugurant la nouvelle ère de la régulation en mettant fin au monopole. Ce serait un euphémisme que de retenir que l'objectif n'a pas été parfaitement atteint. 


\section{La diversification des figures de la dépendance politique}

Dès les premières mesures de mise en œuvre de la réforme, la pérennité des dépendances politiques s'est manifestée sur chacun des deux grands volets de la rupture, qu'il s'agisse du choix des opérateurs privés de télévision, bénéficiaires de la "liberté » de communication, dans le cadre d'un régime de "concessions de service public", aussi bien que des vicissitudes subies par l'autorité dite «indépendante » de régulation.

Le régime des concessions de service public, prévu par la loi de 1982, fut d'abord appliqué à la création de Canal Plus, officiellement, et juridiquement, première chaîne privée créée en novembre 1984. Evoquer le fait que M. André Rousselet, directeur de cabinet du Président de la République, devint, sans transition, P.D-G de cette nouvelle chaîne, pourrait, déjà, constituer un saisissant raccourci de la filiation maintenue entre les deux branches du pouvoir politico-médiatique.

L'application de la loi subséquente du 23 décembre 1985, précisant les modalités de mise en œuvre du régime des concessions de service public pour les nouvelles chaînes privées, de droit commun, se caractérise par la dénaturation des fondements mêmes du régime traditionnel de la concession de service public, dénoncée par la Haute Autorité de la Communication Audiovisuelle, dans un avis du 16 janvier 1986, dépourvu d'effet, au profit d'opérateurs discrétionnairement choisis par le Président de la République sous le prisme du seul calcul politique.

Les capitaines d'industrie, amis politiques du Président - MM. Seydoux et Riboud - firent ainsi alliance avec un certain Berlusconi, naufrageur de la télévision publique italienne pour donner vie à La Cinq, selon un contrat de concession de service public, dépourvu de réel cahier des charges. De telle sorte qu'au terme de ce "coup politique », ce que toute l'opinion profane pouvait découvrir comme la caricaturale manifestation d'une télévision strictement commerciale, parfois même moquée comme «télévision coca-cola » ou « télévision spaghetti ", relevait d'une qualification juridique en termes de service public, ainsi vidée de toute substance. Cette réduction du service public, et de ses missions, à une pure appellation dépourvue de contenu allait peser lourd dans les évolutions ultérieures. La deuxième chaîne privée, TV6, fut également, octroyée, selon la même logique d'affinités politiques électives.

La prévalence des mêmes considérations, proprement politiciennes, dans la ré-attribution des fréquences fut toute aussi manifeste au terme de l'alternance politique consécutive aux élections législatives de 1986. La loi du 30 septembre 1986 supprima le régime des concessions de service public pour lui substituer un régime d'autorisations qui permit de procéder à la résiliation des concessions précédentes, et à l'attribution des fréquences correspondantes aux amis politiques du nouveau Premier Ministre (respectivement emmenés par le Groupe Hersant pour La Cinq, et la Lyonnaise des eaux, dirigée par Jérôme Monod, pour la sixième chaîne, devenue M6). Les chaines précédemment considérées comme concessionnaires de service public devenaient ainsi de simples chaines commerciales privées.

La volonté de maîtrise politique des opérateurs par le Pouvoir Exécutif participe, conjointement, d'une approche centraliste, de nature à éclairer le piteux échec des velléités de décentralisation qui avaient, pourtant, été inscrites dans la loi de 1982: Les "sociétés régionales de radiodiffusion sonores » chargées de gérer les «stations locales de télévision " n'ont jamais vu le jour. Pas davantage que les stations régionales de FR3 n'ont accédé au statut de société que leur promettait la loi dans un délai maximal de quatre ans. Les «comités régionaux de la communication audiovisuelle», dotés d'une compétence consultative auprès de la Haute Autorité, du représentant de l'Etat dans la région, et du 
conseil régional, sont, pareillement, restés à l'état de dispositifs législatifs dépourvus de la moindre effectivité.

Les mêmes vicissitudes politiques entraînèrent les turbulences de l'Autorité de régulation, composée sur la base de choix politiques la rendant soluble dans les échéances électorales. La Haute Autorité de la Communication Audiovisuelle, créée par la loi de 1982, ne résista pas à l'alternance politique de 1986, au terme de laquelle lui fut substituée la CNCL (Commission Nationale pour la Communication et les Libertés). Celle-ci fut, à sont tour, condamnée par la nouvelle alternance politique de 1988, consécutive à la réélection du Président François Mitterrand. De telle sorte que l'autorité de régulation, dite « indépendante », manifestait sa claire dépendance à l'égard des échéances politiques.

La CNCL fournit même une nouvelle forme d'apothéose en termes de filiation politique, conduisant, par exemple, à la nomination, au titre de l'Académie française, du journaliste Michel Droit, intervieweur officiel du Général De Gaulle, et incarnation du modèle de la «Voix de la France » durant la période du monopole public.

Quant à la loi du 17 janvier 1989 consécutive à une nouvelle échéance politique, législative en l'occurrence, son objet même fut étroitement politique : alors que le parti socialiste revenu au pouvoir, ne remettait nullement en cause les fondements libéraux de la loi du 30 septembre 1986 qu'il avait pourtant tellement stipendiée lors de son adoption, la réforme qu'il édictait venait, pour l'essentiel, simplement modifier la loi de 1986 pour substituer à la CNCL une nouvelle autorité, le CSA, reconfiguré sur le modèle initial du Conseil Constitutionnel... De telle sorte que trois autorités de régulation successives avaient vu le jour en moins de sept ans !...

On peut comprendre, dans ce contexte, que le Président Sarkozy, et les membres de sa majorité, aient pu considérer que sur le terrain politique, le CSA ne puisse échapper à une relation de subordination, et qu'ainsi, ses compétences relatives à la nomination des P.D-G de l'audiovisuel public - France-Télévisions, Radio France, Audiovisuel Extérieur relèveraient d'une pure «hypocrisie ». Pour mettre fin à cette - éventuelle - hypocrisie, la loi du 5 mars 2009 transféra donc, directement, ce pouvoir de nomination, et de révocation, au Président de la République, manifestant ainsi au grand jour la filiation maintenue de l'Audiovisuel public avec le Pouvoir Exécutif. L'éventuelle «hypocrisie » fut ainsi reléguée dans le champ procédural, sous forme d'avis conforme du CSA et de l'illusoire garantie prévue par l'article 13 révisé de la Constitution concernant l'intervention des commissions culturelles des deux assemblées pouvant opposer leur véto à ces nominations à la majorité des $3 / 5$.

Mais la loi du 5 mars 2009 s'inscrit aussi, et d'abord, dans la seconde strate archéologique, centrale, de la régulation de la communication audiovisuelle, constituée par les déterminants économiques et financiers.

\section{LE SERVICE PUBLIC CONFRONTE A LA DYNAMIQUE MARCHANDE}

Il convient de souligner, de manière liminaire, que les logiques politiques et les logiques économiques et financières ne sont évidemment pas exclusives l'une de l'autre, même si du fait du monopole public, les secondes n'ont pu pleinement se manifester que plus tardivement.

En réalité, la loi de 1982 elle-même, abolissant le monopole, ne correspondait pas seulement à une logique politique : la revendication des radios dites de "proximité ", ou «alternatives », coincidaient, opportunément, avec les attentes d'importants groupes de pression, agences de publicité et groupes industriels, ayant décelé, depuis longtemps, dans 
le secteur de l'audiovisuel, d'importants gisements de profits, qui ne seront pas déçus par la suite.

Il est à cet égard significatif que les opérateurs de l'économie audiovisuelle aient pu, rapidement, mettre en pièces les dispositifs juridiques constituant initialement, dans la loi de 1982, les conditions de mise en œuvre de la nouvelle liberté de communication: l'ouverture aux radios privées se fondait, à l'origine, sur des conditions telles que l'obligation d'un statut associatif, l'interdiction de ressources publicitaires et l'obligation d'un caractère strictement local. Autant de limites qui allaient, aussitôt, se révéler inaptes à endiguer la pression du marché publicitaire et les objectifs des opérateurs. L'angélisme de la réforme avait été dénoncé par un député de l'opposition de droite, connaissant bien le secteur, Joël Le Tac prévoyant, à la tribune de l'Assemblée Nationale que "Les grands squales carnassiers de la communication audiovisuelle» auraient tôt fait d'avaler «les gentils petits poissons-pilotes » seuls autorisés par la loi de 1982.

Mais la claire prévalence du marché s'imposa, de manière impériale, au terme de l'alternance politique, avec la loi du 30 septembre 1986 procédant à la privatisation de TF1 et à la récusation même de la référence au service public. Cette logique libérale, fût, ensuite, encore approfondie.

\section{Privatisation de TF1 et récusation de la notion de service public}

La loi du 30 septembre 1986 se présente, dans l'archéologie du système audiovisuel français, comme la strate exprimant, toujours, 27 ans plus tard, le cœur du droit positif. Destin paradoxal de ce texte, le plus critiqué, le plus vilipendé lors de son adoption et, contrairement aux lois antécédentes, jamais abrogé !

La privatisation de TF1, première chaine de service public, suffirait à manifester la singularité du modèle français. Ainsi que cela a souvent été relevé, aucun autre Etat européen, même parmi les plus représentatifs des politiques de libéralisation n'avait, jamais privatisé une chaîne publique de télévision. La Grande-Bretagne, même sous la férule de Mme Tchatcher ne proposa pas de privatiser, fût-ce partiellement, la BBC, et la dérégulation italienne, permettant l'avènement de l'Empire audiovisuel privé de Berlusconi, s'est opéré «à côté » du service public de la RAI, et non en se substituant à l'une de ses composantes (8).

Cette situation a été institutionnellement favorisée par la loi antécédente du 7 août 1974, elle-même édictée à la suite d'une alternance politique et l'élection de Valéry Giscard d'Estaing à la présidence de la République. Cette loi, autre singularité française, a procédé au démantèlement de l'organisme unitaire de l'ORTF pour lui substituer sept organismes distincts dont trois sociétés de programme pour la télévision publique, correspondant aux trois chaînes existantes : TF1, Antenne 2 et FR3. La portée de cette loi est très éclairante pour comprendre le devenir ultérieur du service public audiovisuel français :

- d'une part, parce que l'éclatement institutionnel a, sensiblement, facilité la privatisation ultérieure de TF1, selon une logique de "vente par appartement »,

- d'autre part, et surtout, parce qu'elle mettait en place les prémisses de l'ouverture ultérieure au marché. Non seulement les statuts juridiques adoptés pour les trois chaînes de service public, rompant avec la tradition de l'établissement public, étaient ceux de sociétés anonymes, de droit privé, dont le capital était seulement détenu par l'Etat, mais ces trois sociétés étaient placées dans une situation que des spécialistes de la régulation avaient même qualifiée de "concurrence sauvage ", alors même que le monopole public n'avait pas été aboli (9). 
Cette concurrence, sur le double terrain de l'audience et des ressources publicitaires, correspondait au fait que le législateur n'avait conçu aucune modalité de coordination, ou d'harmonisation des programmes entre ces trois sociétés. Situation joliment qualifiée par Jacques Chevallier de «décentralisation sans centre»(10), ou, plus brutalement, par le rapporteur de la loi, au Sénat, de construction «sans cœur, ni tête, ni squelette» (11). Dans ce contexte, le pourcentage des ressources publicitaires dans le budget des chaînes, avait connu une croissance exponentielle, faisant, déjà, du marché publicitaire un facteur central du fonctionnement des chaines de service public, alors que cette ressource commerciale n'avait été introduite qu'à petite dose, supposée devoir telle, après les événements de 1968. La privatisation douze ans plus tard de TF1 concernait ainsi une chaîne - situation unique en Europe - représentant $40 \%$ de l'audience et $55 \%$ du marché publicitaire. Une telle position, quasi-hégémonique, a alors placé TF1 en acteur de la régulation globale du marché, entraînant, en particulier, la faillite de La Cinq, entre-temps détenue par le groupe Hachette.

Complémentairement, la révocation des concessions de service public, précédemment évoquée, constituait la traduction concrète de la récusation même de la qualification de " service public », à l'égard des chaînes publiques résiduelles, seulement appréhendées en termes de "secteur public", qualification ne rendant compte que de la détention de leur capital par l'Etat. On se souvient, à cet égard, de la proclamation du ministre de la Culture et de la Communication, François Léotard, selon laquelle «le service public est mort. Le service public est un astre dont la lumière nous parvient encore mais qui est mort » (12).

La logique de la loi dite Léotard ne fut cependant pas remise en cause par l'alternance suivante, consécutive à la réélection de François Mitterrand. La loi de 1989 - adoptée selon la procédure de l'article 49.3 de la Constitution c'est-à-dire sans vote, à défaut de majorité n'eut pas d'autre objectif que de substituer une nouvelle autorité de régulation à une autre, le CSA à la CNCL. On vit même la ministre chargée de la Communication, Catherine Tasca, s'opposer à un amendement visant à rétablir la qualification de service public, en arguant de ce que la télévision était désormais «une affaire d'entreprise, et non d'administration » (13).

Par la suite, les décrets dits Tasca, au soutien d'une production indépendante, interdisaient la production en interne des chaînes de programmation, s'agissant de la «production lourde » et assuraient à un certain nombre de producteurs privés, une rente de situation, privant corrélativement les programmateurs de droits sur les œuvres diffusées. Les notables dérives d'un tel système d'externalisation de la production au profit de sociétés privées, ont abouti au scandale financier dit des «animateurs-producteurs" bénéficiaires des prodigalités publiques mises au jour par un rapport parlementaire du très libéral député Alain Griotteray, montrant notamment comment une chaine publique avait pu être instrumentalisée pour constituer le capital d'une société de production privée, ensuite bénéficiaire des largesses de la commande publique (14).

Il fallut attendre la loi du $1^{\text {er }}$ août 2000 pour que soit rétablie la référence au service public et aux missions qui la fondent, son article 3 (art. 43.11 de la loi de 1986) indiquant que les sociétés nationales de programme "poursuivent, dans l'intérêt général, des missions de service public». Cette même loi s'appliquait, corrélativement, à rétablir une meilleure cohérence dudit service public en créant une société holding - France-Télévisions regroupant France 2, France 3 et La Cinquième -, la durée de mandat de son président étant porté à 5 ans. Le projet de loi initial avait eu pour fondement de limiter la participation des grands groupes industriels titulaires de marchés publics dans le capital des sociétés privées de télévision. Mais la ferme opposition des groupes concernés avait réduit cette velléité à néant. Entre-Temps, la prévalence des logiques de concurrence et du 
marché audiovisuel s'étaient considérablement confortées dans le contexte des évolutions du droit communautaire.

\section{La menace de délitement du service public}

De manière quasi-concomittante à l'élaboration de la loi précitée de 1974, était intervenue une décision fondamentale de la Cour de Justice des Communautés Européennes, la décision Sacchi du 30 avril 1974 (15) reconnaissant, pour la première fois, que les programmes audiovisuels présentaient la nature de «services" au sens du Traité, et relevaient donc, à ce titre, des principes de libéralisation économique et de librecirculation, inhérents à la construction européenne. Les effets de cette jurisprudence, encore contenus, dans le contexte des monopoles publics nationaux, ont produit leur pleine mesure postérieurement à la fin de ceux-ci, au milieu ou à la fin, des années 1980, en 1986 dans le cas français. Ces effets au regard de la mondialisation du marché de l'audiovisuel ont suscité l'édiction d'une directive européenne, la directive "Télévisions sans frontières » du 3 octobre 1989, entrée en vigueur en 1991(16), mais celle-ci ne faisait, précisément aucune distinction entre télévisions publiques et télévisions privées. Conformément aux principes fondamentaux du droit communautaire, la rationalité de la concurrence ne connait que des entreprises et des opérateurs sur le marché, indépendamment de leur nature publique ou privée. Les enjeux démocratiques du service public ont néanmoins été pris en compte à compter d'un protocole additionnel au Traité d'Amsterdam, en 1997, légitimant les financements publics des chaines de service public. Ces financements sont eux-mêmes conçus de manière restrictive, au regard, notamment, d'un principe de proportionnalité entre leur montant et le coût des obligations de service public.

Dans ce contexte, la loi dite Carignon du $1^{\text {er }}$ février 1994, elle-même violemment stigmatisée par l'opposition de gauche lors de son adoption, mais nullement remise en cause ensuite, notamment après l'alternance de 1997, paraît correspondre à la seule formalisation juridique des attentes et revendications des grands opérateurs privés. Plusieurs mutations importantes en découlent :

Sur le terrain d'abord, des dispositions anti-concentration. Ces dernières avaient, initialement, été oubliées par le législateur de 1986 et la censure du Conseil Constitutionnel, dans sa décision du 18 septembre 1986, fut à l'origine d'une loi complémentaire du 27 novembre 1986, précisément relative à la concentration dans les médias. La loi de 1994 vient précisément, procéder, à un sensible relèvement des seuils anti-concentration. En matière de télévision, le plafond est relevé de $25 \%$ à $49 \%$ de détention du capital. Pour les radios, les seuils ne concernent pas la participation capitalistique mais les bassins d'auditeurs desservis. Le plafond initial de 45 millions (un réseau de 30 millions, n'autorisant qu'un deuxième réseau plafonné à 15 millions) est élevé à 150 millions, soit la maîtrise de trois réseaux nationaux, c'est-à-dire le maximum alors permis par les fréquences hertziennes disponibles.

Plus lourd de conséquences pour la régulation ultérieure, est la mesure qualifiée par les professionnels de "reconduction automatique" des autorisations, hors appel à candidatures : les opérateurs déjà autorisés bénéficient d'un renouvellement pour deux nouvelles périodes de cinq ans, ce qui porte de cinq à quinze ans la durée des autorisations en matière de radios, et de dix à vingt ans leur durée en matière de télévision, conduisant, de fait, à des autorisations « sine die». 


\section{CONCLUSION}

Sous l'éclairage d'une telle analyse archéologique, à bien des égards singulière au regard des autres pays européens, on peut comprendre l'accueil, en apparence, paradoxal, fait à la loi Sarkozy du 5 mars 2009 : la suppression de la publicité commerciale sur les chaînes de service public aurait pu être perçue comme favorable à une meilleure identification et une meilleure qualité des chaînes publiques, de nature à renforcer la légitimité spécifique du service public. Pourtant, le commentaire dominant consista à ne retenir que l'aggravation de la situation financière de France-Télévisions, privée d'une partie non négligeable de ses ressources et dont les contraintes budgétaires sont aujourd'hui particulièrement préoccupantes, au profit d'un effet d'aubaine pour les grands opérateurs privés destinés à récupérer la manne financière perdue par le service public.

La réforme de 2009 fut appréhendée à travers le double prisme, politique, de la nomination des P-D.G de l'audiovisuel public par le Président de la République et financier, de la suppression des ressources publicitaires. En revanche, un autre aspect de la réforme consistant à réunifier l'organisation du service public en une société unique fut largement passé sous silence alors qu'il venait, opportunément, corriger les avatars consécutifs au démantèlement de l'organisme unitaire de l'ORTF, trente-cinq ans plus tôt (17).

Pourtant, dans le même temps, la montée en puissance d'Internet, le développement plus rapide et plus important que prévu des chaînes du Numérique Terrestre, ont superposé sur les déterminantes antécédentes, une nouvelle sédimentation de déterminantes techniques, ou technologiques. Ces déterminantes technologiques n'ont, certes pas, surgi ex-nihilo. Elles dominent le débat public depuis plusieurs années mais elles ont, longtemps, relevé d'un argument marketing pour les opérateurs privés, utilisant l'argument du déterminisme technologique comme un "cheval de Troie » pour accélérer les processus de libéralisation du système audiovisuel.

Au cours des années récentes, ces mutations techniques apparaissent, en revanche, au cœur de modifications sensibles des usages sociaux, des pratiques sociales, de nature à produire de nouvelles et profondes ruptures, en forme de remise en cause de la définition classique des chaînes de télévision elles-mêmes au profit de plate-formes numériques organisées sur la base des choix de programmation partiellement pilotés par les consommateurs. Dans plusieurs pays européens, comme la Grande-Bretagne, se fait jour la thèse selon laquelle la télévision devrait échapper à une perception organique en termes de chaines publiques opposées aux chaines privées, et que devrait, à l'inverse, prévaloir une conception fonctionnelle en termes de missions de service public qui pourraient, indifféremment, être confiées aux différentes chaines.

Ces nouveaux enjeux, et nouvelles couches archéologiques encore ignorées par les lois en cours de discussion, sont, déjà, au cœur des interpellations contemporaines relatives à l'avenir du service public audiovisuel français. 


\section{REFERENCES}

1. CE 11 février 2010, Mme Borvo et autres, chronique S.J Lieber et D. Botteghi, AJDA 2010, p. 670 .

2. Regourd, Serge, (2008), Vers la fin de la télévision publique? Traité de savoir-vivre du service public audiovisuel, Ed. de l'Attribut, p.53.

3. Foucault, Michel, (1969), L'archéologie du savoir. Gallimard.

4. C.Cons. 3 mars 2009, n²009-577 DC.

5. Regourd, Serge, (2001), Droit de la communication audiovisuelle. PUF, collect. Droit fondamental, p.58 et s.

6. Regourd, Serge, (1983), "Le droit de la communication audiovisuelle après la loi du 29 juillet 1982", Actualité législative Dalloz, p.23.

7. Mac-Luhan, Harold M., (1968), Pour comprendre les médias, Le Seuil, collect. Points.

8. Regourd, Serge, (1992), La télévision des européens. La Documentation française, collect. Vivre en Europe, p.248 et s.

Musso, Pierre et Pineau, Guy, (1989), L'Italie et sa télévision, INA/Champ Vallon.

9. Guillou, Bernard et Padioleau, Jean-Gustave (1988), La régulation de la télévision, La Documentation française.

10. Chevallier, Jacques, (1982), "Le statut de la communication audiovisuelle", AJDA, p. 555.

11. Rapport Miroudot, J.O Doc. Senat, $\mathrm{n}^{\circ} 288$, p.18.

12. F. Leotard, Sénat, 26 juin 1986 J.O Débats, p.2013.

13. C .Tasca, A.N 9 mai 1989 J.O Débats, p.728.

14. Regourd, Serge, (1997), "De la crise comme élément d'identification du service public français de l'audiovisuel", Sciences de la Société, n 42 , p.75.

15. CJCE Aff.155/73 30 avril 1974, Rec. p. 409.

16. "Directive $\mathrm{n}^{\circ} 552$ du 3 octobre 1989 visant la coordination de certaines dispositions législatives, réglementaires et administratives des Etats membres relatives à l'exercice d'activités de radiodiffusion audiovisuelle", JOCE 17 octobre 1989 n² 298, p. 23.

17. Regourd, Serge, (2009), "La loi du 5 mars 2009 et le nouveau service public de la télévision : une régénération controversée", Légipresse, n²60, p. 29. 